\title{
A case of Behçet disease with pulmonary artery pseudoaneurysm: long term follow-up
}

\author{
M-H.S. Modaghegh, ${ }^{7}$ G. H. Kazemzadeh ${ }^{7}$ and M. H. Jokar ${ }^{2}$
}

\section{Introduction}

Behçet disease (BD) is a chronic inflammatory disorder of unknown etiology with a clinical spectrum that has greatly expanded since it was first described in 1937 by Hulusi Behçet as a triple complex of recurrent oral and genital ulcers and uveitis [1].

Although BD has a worldwide distribution, it is mainly seen in Far East and Middle East countries [2,3]. The highest prevalence is reported from Turkey, 80-370 per 100000 , and ranges from 2 to 30 cases per 100000 in other Asian countries (16.7 per 100000 in the Islamic Republic of Iran) $[2,3]$. The age of disease onset is usually in the second or third decade of life and the male to female ratio is reported to be almost equal. However, the disease runs a more severe course in men and in those with onset before 25 years of age [2].

Since BD does not have pathognomonic symptoms or laboratory findings; diagnosis is made on the basis of the criteria proposed by the International Study Group for Behçet disease in 1990 [4]. According to the criteria, recurrent oral ulceration must be present along with at least 2 of the following: recurrent genital ulceration, eye lesions, skin lesions, a positive pathergy test $[2,4,5]$. Many disorders, such as cutaneous small vessel vasculitis, inflammatory eye disease, neurological disease, vascular disease, arthritis, are associated with the presence of BD [6]. Pathological features such as small- or large-vessel vasculitis lead to occlusion of the vessels or aneurysm formation [7]. Differential diagnosis of Behçet-induced vasculitis has been described by Kreuger, Hoffman and Merton [8].

More than 200 cases of BD with pulmonary involvement have been reported in the literature. The pulmonary arteries are the second most common site of arterial involvement after the aorta. Aneurysms are more common than thrombosis [2]. Aneurysms associated with $\mathrm{BD}$ tend to be multiple and pulmonary artery aneurysms are relatively common, with an associated rate of $1 \%-10 \%$ of patients with BD [9]. Haemoptysis of varying degrees (up to 500 $\mathrm{mL}$ ) is the most common and predominant symptom [2]. Medical treatment is more beneficial when given in the early stage of the disease [10]. Surgical repair of pulmonary artery aneurysm in $\mathrm{BD}$ carries a high risk, with high rates of morbidity and mortality [11]. Recently, endovascular management has been attempted for treatment of vascular involvement. However, when aneurysms are multiple, endovascular treatment is still difficult and challenging.

To our knowledge, there is no report on a patient with 4 or more arterial aneurysms treated solely by endovascular management $[12,13]$.

\section{Case report}

A 32-year-old woman was admitted to the hospital with painful oral and genital ulcerations, unilateral oedema of the lower limb, blurred vision and polyarthralgia. On physical examination vital signs were stable. Respiratory sounds were decreased in the left lung. Laboratory tests revealed erythrocyte sedimentation rate $=152 \mathrm{~mm} / \mathrm{h}$ and anaemia. At the age of 22 years, following amnesia for 19 days, she had been referred to a neurologist and examination revealed bilateral papillary oedema and raised intracranial pressure. The patient was given oral acetazolamide, $125 \mathrm{mg}$ twice a day.

Regarding her past medical history, ophthalmologic consultation was performed and a mild retinal vasculitis was found via ophthalmoscopy. Extensive deep vein thrombosis extending to the inferior vena cava was demonstrated in colour Doppler study. According to the international criteria for diagnosis [4], she was diagnosed as having BD (recurrent mouth and genital aphthous ulcers and eye lesion). She was prescribed 750 mg pulse cyclophosphamide monthly, with warfarin $2.5 \mathrm{mg} /$ day and methyl prednisolone $1 \mathrm{~g} /$ day for 3 days, followed by oral prednisolone $1 \mathrm{mg} / \mathrm{kg}$ per day, tapered gradually and continued at a low dose. Two weeks later, oedema of the lower limb had completely resolved, thrombus size had substantially decreased and the patient was discharged in good condition.

After 1 year, she was admitted again to the internal medicine ward, with a 5-month history ofdyspnoea, cough and haemoptysis. Purified protein derivative 


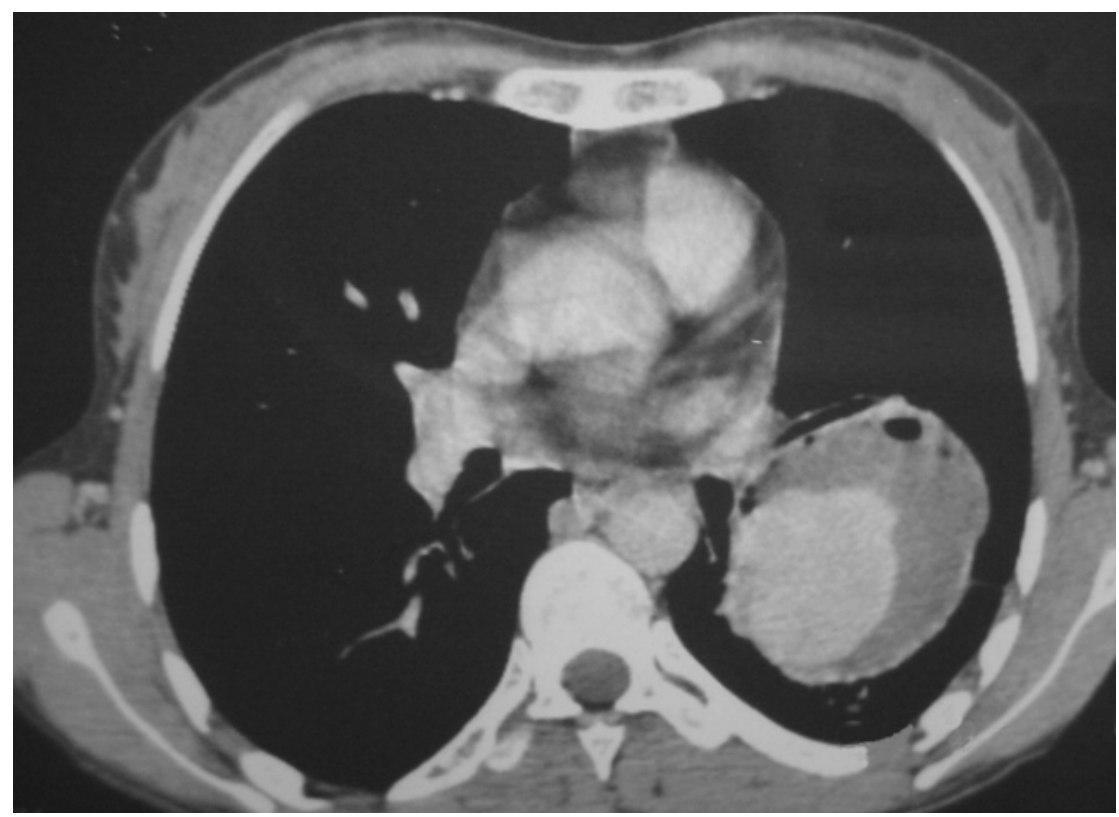

Figure 1 Computerized tomography scan of the thorax with contrast showing a large cavity at the inferior lobe with extension to the hill and adhesion to the chest wall, indicating a large pulmonary artery aneurysm

test and sputum smear were performed 3 times but the results were negative. No history of recent or remote trauma, drug abuse or infective endocarditis was elicited. On examination, the patient appeared chronically ill and pale; her blood pressure was 100/70 mmHg and pulse rate was $70 / \mathrm{min}$; renal and liver function tests were normal; S2 splitting was detected on chest examination; oral and genital lesions were noted. White blood count was $8.06 \times 10^{3} / \mu \mathrm{L}$, haemoglobin was $10.0 \mathrm{~g} / \mathrm{dL}$, haematocrit was $32.1 \%$ and erythrocyte sedimentation rate was $41 \mathrm{~mm} / \mathrm{h}$.

Chest X-ray showed a $6 \times 6 \mathrm{~cm}$ opacity in the inferior lobe of the left lung. Computerized tomography (CT) scan of the thorax with intravenous contrast revealed an enhanced large cavity at the inferior lobe with extension to the hill and adhesion to the chest wall indicating a large pulmonary artery aneurysm (Figure 1). The diagnosis was further confirmed by a digital subtraction angiography (Figure 2). The patient started to receive $1 \mathrm{mg} / \mathrm{kg}$ prednisone daily and $750 \mathrm{mg}$ cyclophosphamide intravenously. After 17 days, she experienced into consideration her previous imaging, we decided to perform elective surgery. Three days later, the patient felt better and suffered less from haemoptysis.

Under general anaesthesia with 1 lung intubation, left thoracotomy was performed. The upper lobe was collapsed and the lower lobe remained expanded because of the contained aneurysm. The left pulmonary artery was proximally controlled at the origin. Further exploration revealed a $10 \times 10$ $\mathrm{cm}$ aneurysm contained in the lower lobe and intact upper lobe branch. The aneurysm was opened. All lower lobe branches of the pulmonary artery were involved in the aneurysm. Resection of the lower lobe was performed and inflation of the upper lobe was controlled before closure.

The gross pathological examination of the specimen revealed a pseudoaneurysm at the lower lobe in addition to bleeding into the adjacent lung parenchyma. Further histopathological evaluation revealed focal coagulation necrosis in addition to neutrophil-cell reaction in the wall of the pulmonary

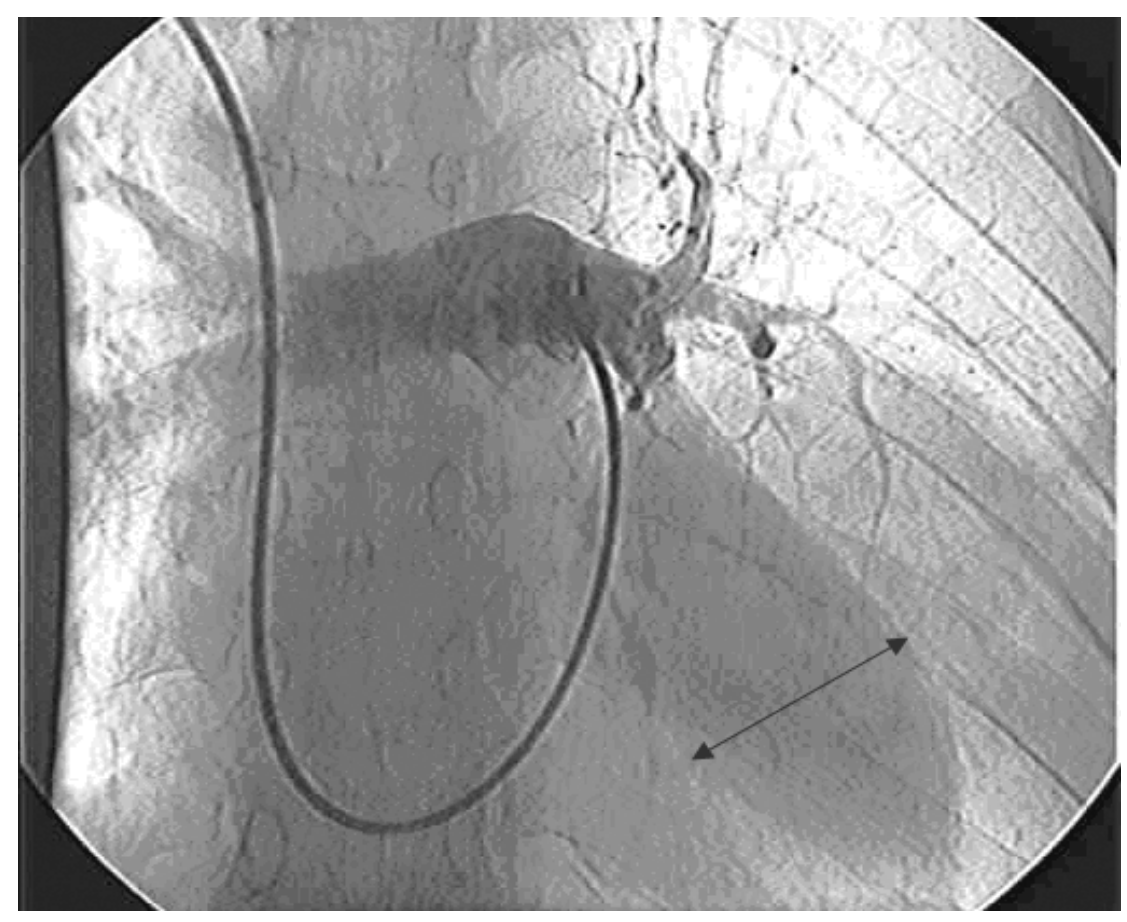

Figure 2 Digital subtraction angiography showing a cavity at the pulmonary artery 
artery. Focal haemorrhagic necrosis and lymphoplasmocytic inflammatory reaction were detected in the lung parenchyma.

The patient was discharged in good condition after 36 days. She has been under close observation for 50 months without suffering relapse of the symptoms or new complaints (Figure 3).

\section{Discussion}

BD is a systemic vasculitis affecting virtually all types and sizes of vessels. Pulmonary manifestations in patients are mainly related to vasculitis of the pulmonary arteries, veins and septal capillaries. Pulmonary vascular involvement can lead to aneurysm formation (pulmonary artery aneurysm), thrombotic occlusion, mainly in the vena cava, pulmonary infarction and pulmonary haemorrhage. Venous system involvement is more common than arterial system involvement; however, rupture of an arterial aneurysm remains a major cause of mortality related to BD. Rupture of an aneurysm with erosion into a bronchus and the development of in situ thrombosis from active vasculitis have been suggested as explanations for the haemoptysis. Sudden hilar enlargement or the appearance of polylobular and round opacities on the chest radiograph can represent pulmonary artery aneurysms. As in our case, the most common symptom of pulmonary arterial aneurysm is haemoptysis.

Medical treatment with immunosuppressive drugs alone or in

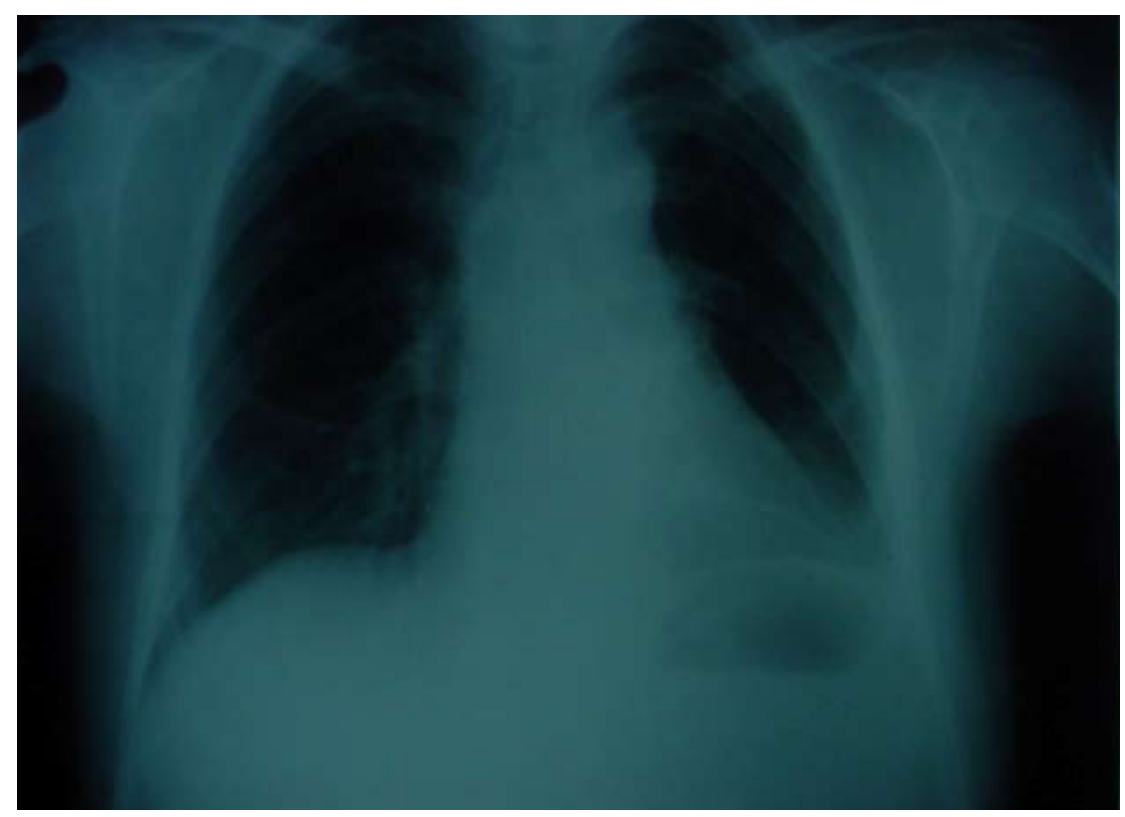

Figure 3 Chest X-ray showing no abnormality 2 months after surgery

combination with steroids is most beneficial when given in the early stage of the disease before irreversible damage to the arterial wall develops [7]. Surgery is available for resecting arterial aneurysms and replacing grafts. Many attempts have been made to manage these serious lesions by surgical treatment. Despite several reports concerning recurrence after surgical management in about half of cases, our patient had no recurrent haemoptysis, productive cough or aneurysm in 50 months of follow up $[13,14]$.

As in our patient, lung lobectomy has been reported in several articles [15-18]. In our patient, the upper lobe of the left lung was intact and all of the lower lobe branches arose from the aneurysm sac. Therefore considering the special characteristic of the aneurysm and the high risk of rupture, resection was performed. The patient was discharged 36 days after the operation, which was much longer than the expected hospitalization period; this was due to prolonged moderate air leakage from the chest tube, which was eventually sealed. Although some articles mention fatal outcomes after lobectomy $[15,17]$, our surgery was completely successful and had a satisfactory result after a long term followup.

In conclusion, pulmonary lobectomy can be performed safely as a treatment for pulmonary aneurysm in $\mathrm{BD}$.

\section{References}

1. Hammami S et al. Intracardiac thrombus in Behçet's disease: two case reports. Thrombosis journal, 2005, 3:9.

2. Erkan F, Gül A, Tasali E. Pulmonary manifestations of Behçet's disease. Thorax, 2001, 56(7):572-8.

3. Davatchi F et al. Behçet's disease-Analysis of 3443 cases. APLAR journal of rheumatology, 1997, 1:2-5.

4. International study group for Behçet's disease. Criteria for diagnosis of Behçet's disease. Lancet, 1990, 335(8697):1078-80.
5. Uzun O, Akpolat T, Erkan L. Pulmonary vasculitis in Behçet disease. Chest, 2005, 127(6):2243-53.

6. Barnes CG, Yazici H. Behçet's syndrome. Rheumatology, 1999, 38(12):1171-4.

7. Çil BE et al. Embolization of a giant pulmonary artery aneurysm from Behçet disease with use of cyanoacrylate and the Bubble technique. Journal of vascular and interventional radiology, 2005, 16(11):1545-9. 
8. Krueger GRF, Hoffmann A, Merten UP. Systemic vasculitis, primary and secondary differential diagnosis and classification. WASPaLM Global Pathology Supercourse, 2000 [internet monograph] (http://www.dokkyomed.ac.jp/dep-k/cli-path/ a-super/vasculitis/super-vas.html, accessed 09 August 2009).

9. Gebitekin $\mathrm{C}$ et al. Fatal hemoptysis due to pulmonary artery aneurysm in Behçet's disease. European journal of vascular \& endovascular surgery, 1997, 13(2):233-6.

10. Tunaci $\mathrm{M}$ et al. CT findings of pulmonary artery aneurysms during treatment for Behçet's disease. American journal of roentgenology, 1999, 172(3):729-33.

11. Bozkurt AK. Embolization in Behçet's disease. Thorax, 2002, 57(5):469-70.

12. Cantasdemir M et al. Emergency endovascular management of pulmonary artery aneurysms in Behçet's disease: report of two cases and a review of the literature. Cardiovascular and intervention al radiology, 2002, 25(6):533-7.

13. Hama $\mathrm{Y}$ et al. Endovascular management of multiple arterial aneurysms in Behçet's disease. British journal of radiology, 2004, 77(919):615-9.
14. Okada K et al. Surgical management of Behçet's aortitis: a report of eight patients. Annals of thoracic surgery, 1997, 64(1):116-9.

15. Tuzun $\mathrm{H}$ et al. Surgical therapy of pulmonary arterial aneurysms in Behçet's syndrome. Annals of thoracic surgery, 1996, 61(2):733-5.

16. Ceyran H, Akcali Y, Kahraman C. Surgical treatment of vasculo-Behçet's disease: a review of patients with concomitant multiple aneurysms and venous lesions. Vasa, 2003, 32(3):149-53.

17. Yassine $\mathrm{N}$ et al. Anevrysmes e l'artere pulmonaire au cours de la maladie de Behçet. A propos de 5 nouvelles observations [Aneurysms of the pulmonary artery in Behçet disease. Apropos of 5 new cases]. Revue de pneumologie clinique, 1997, 53(1):42-8.

18. Salamon F et al. Massive hemoptysis complicating Behçet's syndrome: the importance of early pulmonary angiography and operation. Annals of thoracic surgery, 1988, 45(5):566-7.

\section{Eastern Mediterranean Approach to Noncommunicable Diseases}

Chronic diseases such as cardiovascular, diabetes, cancer, renal, genetic and respiratory conditions are rising dramatically in the Eastern Mediterranean Region. Currently, $45 \%$ of the region's disease burden is due to noncommunicable diseases. It is expected that this burden will rise to $60 \%$ by the year 2020 . The impact of these conditions falls heavily on the region's poor and marginalized populations.

The Eastern Mediterranean Approach to Noncommunicable Diseases has the overall objective to improve the health of populations of the Region by reducing mortality and morbidity from major NCDs through an integrated collaborative intervention programme of prevention and health promotion.

Further information about the work of EMRO in tackling noncommunicable diseases s is available at: http:/ /www. emro.who.int/ncd/ncd_introduction.htm 